\section{An Optimal Preview Control for Linear Systems}

\section{C. MacAdam}

A technique for synthesizing closed-loop control of linear time-invariant systems during tracking of previewed inputs is presented. The derived control is directly dependent upon the properties of the controlled system and is obtained by minimization of a defined previewed output error.

\section{Introduction}

This paper presents a general method of control synthesis applicable to linear time-invariant systems utilizing preview control strategies for regulation or tracking tasks. A common example of this type of dynamical behavior occurs during normal automobile path following in which drivers "lookahead" to follow a desired path. A frequent source of preview control strategies in various man-machine systems is, of course, the human operator. It is widely recognized that human operators are capable of controlling and adapting to a wide variety of dynamical systems, many of which are vehicles with preview-oriented control requirements such as automobiles, bicycles, and complex aircraft [1-7]. Although this paper does not offer evidence as to the utility of the proposed control synthesis for man-machine systems involving preview strategies, it is suggested that the method presented here can be applied to such problems. Portions of the work by Tomizuka [8], which treated a similar problem, indicated useful application of optimal preview control methods in representing man-machine dynamical behavior.

The particular method presented in this paper is directly applicable to general linear system representations assumed to incorporate preview control strategies that depend only upon knowledge of the current values of the state and control. The optimal control is derived by minimization of a performance index that is defined as a mean squared preview output error. It will be shown that the derived control function is not arbitrary or independent but depends directly upon the dynamical properties of the controlled system.

\section{Statement of the Problem}

Given the linear system

$$
\begin{gathered}
\dot{\mathbf{x}}=F \mathbf{x}+\mathbf{g} u \\
y=\mathbf{m}^{T} \mathbf{x}
\end{gathered}
$$

\footnotetext{
${ }^{1}$ Research Associate, University of Michigan, Highway Safety Research Institute, Ann Arbor, Mich. 48109

Contributed by the Dynamic Systems and Control Division of THE AMERICAN SOCIETY OF Mechanical Engineers. Manuscript received at ASME Headquarters, July 9, 1980
}

where,

$\mathbf{x}$ is the $n \times 1$ state vector

$y$ is the scalar output related to the state by the $n \times 1 \mathbf{m}^{T}$ constant observer vector transpose

$F$ is the constant $n \times n$ system matrix

and

$\mathrm{g}$ is the constant $n \times 1$ control coefficient vector

find the control, $u(t)$, which minimizes a local performance index,

$$
J \triangleq \frac{1}{T} \int_{t}^{t+T}\{[f(\eta)-y(\eta)] W(\eta-t)\}^{2} d \eta
$$

over the current preview interval $(t, t+T)$, where,

$W$ is an arbitrary weighting function over the preview interval

and $f$ is the previewed input.

The performance index given by (3) represents the weighted mean squared error between the previewed input and the previewed output as defined below.

The previewed output, $y(\eta)$, is related to the present state, $\mathbf{x}(t)$, by

$$
y(\eta)=\mathbf{m}^{T} \phi(\eta, t) \mathbf{x}(t)+\int_{l}^{\eta} \mathbf{m}^{T} \phi(\eta, \xi) \mathbf{g} u(\xi) d \xi
$$

where,

$$
\phi(\eta, t)=\exp [F(\eta-t)]
$$

is the transition matrix of the system $F$ [9].

If $u(t)$ is assumed selected on the basis of a constant previewed control, $u(\xi)=u(t)$, equation (4) simplifies to

$$
y(\eta)=\mathbf{m}^{T} \phi(\eta, t) \mathbf{x}(t)+u(t) \int_{t}^{\eta} \mathbf{m}^{T} \phi(\eta, \xi) \mathbf{g} d \xi
$$

and the performance index, (3), can be written as

$$
\begin{aligned}
& J=\frac{1}{T} \int_{t}^{t+T}\left\{\left[f(\eta)-\mathbf{m}^{T} \phi(\eta, t) \mathbf{x}(t)\right.\right. \\
&\left.\left.\quad-u(t) \int_{t}^{\eta} \mathbf{m}^{T} \phi(\eta, \xi) \mathbf{g} d \xi\right] \cdot W(\eta-t)\right\}^{2} d \eta
\end{aligned}
$$

The above assumption simply requires the resulting optimization to reflect a control strategy dependent only upon current values of the state and control. This assumption is, in part, motivated by the potential application to those manmachine systems, wherein, it is assumed the human operator is limited in deriving or having knowledge a priori of more complex or optimal control waveforms over the preview interval.

The necessary condition for minimization of $J$, defined by 
equation (6), with respect to the control, $u(t)$, is provided by $d J / d u=0$, or

$$
\begin{aligned}
\frac{d J}{d u}=\frac{2}{T} \int_{t}^{t+T}\left\{\left[f(\eta)-\mathbf{m}^{T} \phi(\eta, t) \mathbf{x}(t)\right.\right. \\
\left.\left.-u(t) \int_{t}^{\eta} \mathbf{m}^{T} \phi(\eta, \xi) \mathbf{g} d \xi\right]\right\} \\
\cdot\left\{\int_{t}^{\eta} \mathbf{m}^{T} \phi(\eta, \xi) \mathbf{g} d \xi\right\} W(\eta-t) d \eta=0
\end{aligned}
$$

Equating $\phi(\eta, \xi)$ with $\exp [F(\eta-\xi)]=\mathrm{I}+\sum_{n=1}^{\infty} F^{n} \frac{(\eta-\xi)^{n}}{n !}$, where $I$ is the identity matrix, and performing the $d \xi$ integrations, (7) becomes

$$
\begin{gathered}
\frac{d J}{d u}=\frac{2}{T} \int_{t}^{t+T}\left\{f(\eta)-\mathbf{m}^{T}\left[I+\sum_{n=1}^{\infty} \frac{F^{n}(\eta-t)^{n}}{n !}\right] \mathbf{x}(t)\right. \\
\left.-(\eta-t) \mathbf{m}^{T}\left[I+\sum_{n=1}^{\infty} \frac{F^{n}(\eta-t)^{n}}{(n+1) !}\right] \mathbf{g} u(t)\right\}\left\{(\eta-t) \mathbf{m}^{T}\right. \\
\left.\cdot\left[I+\sum_{n=1}^{\infty} \frac{F^{n}(\eta-t)^{n}}{(n+1) !}\right] \mathbf{g}\right\} W(\eta-t) d \eta=0
\end{gathered}
$$

Solving (8) for $u(t)$ yields

$$
\begin{aligned}
& u^{0}(t)=\left[\int_{t}^{t+T}\left\{f(\eta)-\mathbf{m}^{T}\left[I+\sum_{n=1}^{\infty} \frac{F^{n}(\eta-t)^{n}}{n !}\right] \mathbf{x}(t)\right\}\right. \\
& \left.\cdot\left\{(\eta-t) \mathbf{m}^{T}\left[I+\sum_{n=1}^{\infty} \frac{F^{n}(\eta-t)^{n}}{(n+1) !}\right] \mathbf{g}\right\} W(\eta-t) d \eta\right] \\
& /\left[\int_{t}^{t+T}\left\{(\eta-t) \mathbf{m}^{T}\left[I+\sum_{n=1}^{\infty} \frac{F^{n}(\eta-t)^{n}}{(n+1) !}\right] \mathbf{g}\right\}^{2} W(\eta-t) d \eta\right]
\end{aligned}
$$

where $u^{0}(t)$ represents the optimal solution. For the special case of $W(\eta-t)=\delta\left(T^{*}\right)$, the Dirac delta function for 0 $<T^{*} \leq T$, (9) simplifies to

$$
\begin{aligned}
u^{0}(t) & =\frac{f\left(t+T^{*}\right)-\mathbf{m}^{T}\left[I+\sum_{n=1}^{\infty} \frac{F^{n}\left(T^{*}\right)^{n}}{n !}\right] \mathbf{x}(t)}{T^{*} \mathbf{m}^{T}\left[I+\sum_{n=1}^{\infty} \frac{F^{n}\left(T^{*}\right)^{n}}{(n+1) !}\right] \mathbf{g}} \\
= & {\left[f\left(t+T^{*}\right)-y_{0}\left(t+T^{*}\right)\right] /\left(T^{*} K\right) }
\end{aligned}
$$

where

$$
K \triangleq \mathbf{m}^{T}\left[I+\sum_{n=1}^{\infty} \frac{F^{n}\left(T^{*}\right)^{n}}{(n+1) !}\right] \mathbf{g} .
$$

Equation (11) represents a proportional controller with gain inversely related to the preview interval, $T^{*}$, and operating on the error between the previewed input, $f\left(t+T^{*}\right)$, and $y_{0}\left(t+T^{*}\right)$, that portion of the previewed output deriving from the state vector's current initial condition. Likewise, equation (9) can be interpreted as a proportional controller operating on a similar error averaged and weighted over the preview interval $(t, t+T)$ by the additional terms appearing in equation (9).

The optimal solution, $u^{0}(t)$, can also be expressed in terms of any current non-optimal $u(t)$ and correspondingly nonzero preview output error, $\epsilon(t)$, by writing equation (9) as

$$
\begin{gathered}
u^{0}(t)=\left[\int_{t}^{t+T}\left\{f(\eta)-\mathbf{m}^{T} \phi(\eta, t) \mathbf{x}(t)-u(t) A(\eta)\right\}\right. \\
\left.\cdot A(\eta) W(\eta-t) d \eta+u(t) \int_{t}^{t+T} A^{2}(\eta) W(\eta-t) d \eta\right] \\
/\left[\int_{t}^{t+T} A^{2}(\eta) W(\eta-t) d \eta\right]
\end{gathered}
$$

or

$$
u^{0}(t)=u(t)+\frac{\int_{t}^{t+T} \epsilon(\eta) A(\eta) W(\eta-t) d \eta}{\int_{t}^{t+T} A^{2}(\eta) W(\eta-t) d \eta}
$$

where

$$
\begin{gathered}
A(\eta) \triangleq(\eta-t) \mathbf{m}^{T}\left[I+\sum_{n=1}^{\infty} \frac{F^{n}(\eta-t)^{n}}{(n+1) !}\right] \mathbf{g} \\
\epsilon(\eta) \triangleq f(\eta)-\mathbf{m}^{T} \phi(\eta, t) \mathbf{x}(t)-u(t) A(\eta) \\
\phi(\eta, t) \triangleq \mathbf{I}+\sum_{n=1}^{\infty} \frac{F^{n}(\eta-t)^{n}}{n !}
\end{gathered}
$$

For the special case of $W(\eta-t)=\delta\left(T^{*}\right)$, as before, equation (13) reduces to

$$
u^{0}(t)=u(t)+\frac{\epsilon\left(t+T^{*}\right)}{T^{*} \cdot K}
$$

The formulation expressed by equation (13) can be useful in describing systems which do not achieve, though closely approximate, the optimal system behavior. Such cases may arise from limitations in achieving the precise optimal control due to time lags or dynamic properties inherent in the controller and not accounted for a priori in the optimization.

While equations (9) and (13) are equivalent mathematically, the latter deomonstrates an explicit relationship between the derived optimal control and the previewed output error function appearing in the performance index of the original problem formulation. Simply stated, the current control level is modified only in response to a nonzero function of the previewed output error, and, in this sense, analogous to an integral controller.

Finally, dependence of the derived optimal control upon the system $(F, \mathbf{g})$ properties is clearly demonstrated by the explicit presence of $F$ and $\mathbf{g}$ in equations (9) and (13). Furthermore, information concerning stability of the closed-loop system utilizing the optimal preview control of equation (9) or (13) is provided by the characteristic roots of the constant matrix

$$
F-\frac{\mathbf{g m}^{T} \int_{0}^{T}\left\{\left[I+\sum_{n=1}^{\infty} \frac{F^{n}(\eta)^{n}}{n !}\right]\right\}\left\{\eta \mathbf{m}^{T}\left[I+\sum_{n=1}^{\infty} \frac{F^{n}(\eta)^{n}}{(n+1) !}\right] \mathbf{g}\right\} W(\eta) d \eta}{\int_{0}^{T}\left\{\eta \mathbf{m}^{T}\left[I+\sum_{n=1}^{\infty} \frac{F^{n}(\eta)^{n}}{(n+1) !}\right] \mathbf{g}\right\}^{2} W(\eta) d \eta}
$$


or

$$
\left[F-\mathrm{ge}^{T}\right]
$$

where

$$
\mathbf{c}^{T}=\frac{\mathbf{m}^{T} \int_{0}^{T}\left\{\left[I+\sum_{n=1}^{\infty} \frac{F^{n}(\eta)^{n}}{n !}\right]\right\}\left\{\eta \mathbf{m}^{T}\left[I+\sum_{n=1}^{\infty} \frac{F^{n}(\eta)^{n}}{(n+1) !}\right] \mathbf{g}\right\} W(\eta) d \eta}{\int_{0}^{T}\left\{\eta \mathbf{m}^{T}\left[I+\sum_{n=1}^{\infty} \frac{F^{n}(\eta)^{n}}{(n+1) !}\right] \mathbf{g}\right\}^{2} W(\eta) d \eta}
$$

resulting from the substitution of (9) into (1). For the special case of $W(\eta)=\delta\left(T^{*}\right),(15)$ becomes

$$
F-\left\{\mathbf{g m}^{T}\left[I+\sum_{n=1}^{\infty} \frac{F^{n}\left(T^{*}\right)^{n}}{n !}\right] /\left(T^{*} \cdot K\right)\right\}
$$

\section{Summary}

The optimal preview control model presented here offers a useful and direct method for representing closed-loop behavior of linear systems utilizing preview control strategies. The derived control is directly related to the properties of the linear system and the previewed input. Further, the method is formulated in terms of general linear system representations, thereby permitting applications to a wide variety of problems.

\section{References}

1 McRuer, D.T., et al., "New Approaches to Human-Pilot/Vehicle Analysis," Systems Technology, Inc., Tech. Rept. AFFDL-TR-67-150, Feb. 1968

2 Wierwille, W.W., Gagne, G.A., and Knight, J.R., "An Experimental Study of Human Operator Models and Closed-Loop Analysis Methods for High-Speed Automobile Driving," IEEE Trans, on Human Factors in Electronics, Vol. HFE-8, No. 3, Sept. 1967, pp. 187-201.

3 Tanaka, K., Goto, N., and Washizu, K., "A Comparison of Techniques for Identifying Human Operator Dynamics Utilizing Time Series Analysis," Proceedings of the Twelfth Annual Conference on Manual Control, University of Illinois, Urbana, Ill., May 25-27, 1976, pp. 673-693.

4 Weir, D.H., "Motorcycle Handling Dynamics and Rider Control and the Effect of Design Configuration on Response and Performance," Ph.D. thesis, University of California, Los Angeles, 1972.

5 Ben-Ari, S., and Ellis, J.R., "The Control of an Articulated Semitrailer Vehicle," Vehicle Safety Legislation-Its Engineering and Social Implications, Mechanical Engineering Publications Limited, London, 1975.

6 Kleinman, D.L., Baron, S., and Levison, W.H., "An Optimal Control Model of Human Response, Part I: Theory and Validation," Automatica, Vol. 6, 1970, pp. 357-369.

7 McRuer, D.T., et al., "New Results in Driver Steering Control Models," Human Factors, Vol. 19, Aug. 1977, pp. 381-397.

8 Tomizuka, M., "The Optimal Finite Preview Problem and Its Application to Man-Machine Systems," Dissertation, MIT, Cambridge, Mass., Sept., 1973.

9 D'Angelo, H., Linear Time-Varying Systems: Analysis and Synthesis, Allyn and Bacon, Boston, 1970.

\section{Asymptotic Theory of Freight Car Hunting}

\section{A. M. Whitman ${ }^{1}$}

A simple formula is derived for the hunting speed of a freight car from an 8 degree of freedom linear model using asymptotic techniques. A comparison is made between the approximation and exact (numerical) solutions. The two agree within 10 percent for parameter values typical of present designs.

\footnotetext{
${ }^{1}$ Director of Research, Railroad Dynamics, Inc., Ardmore, Pa.

Present Address: Associate Professor, University of Pennsylvania, Philadelphia, $\mathrm{Pa}$.

Contributed by the Dynamic Systems and Control Division of THE AMERICAN Society of Mechanical EngINeERs. Manuscript received at ASME Headquarters, July 9, 1980.
}

\section{Introduction}

The purpose of the present paper is twofold. The first is to obtain an analytic expression for the critical speed of a multidegree of freedom model of a freight car which is simple enough to convey physical insight into the hunting problem while at the same time complex enough to have validity for realistic vehicles. The second is to illustrate the simplification which can be effected in problems of this type by employing asymptotic methods. These methods are model independent and rely on the fact that the creep forces dominate the motion.

Previous work has included analytical studies of simple vehicles [1-2] and numerical solutions for realistic vehicles [34]. The present work can be viewed as a generalization and formal mathematical justification of the former, which although cleverly done are ad hoc by nature and seem to be restricted to systems with few degrees of freedom, and a specialization of the latter, giving the same results in the region of validity of the expansion but being restricted by nature to specific regions in parameter space. The utility of the present work is in the simple result which it yields. From this one can obtain physical insight into the phenomenon as well as easily calculable answers.

\section{Model Description}

We consider a model of the lateral dynamics of a freight car composed of a rigid car body pinned at either end to a truck. The pin connection transmits a linear damping moment (constant $c^{\prime}{ }_{f}$ ) between the car body and the truck. Each truck, see Fig. 1, is composed of 2 wheelsets, two rigid sideframes connected by ball joints to each wheelset, and a bolster, which contains the car connection (centerplate) at its midpoint, is constrained to move parallel to each wheelset by means of frictionless slotted pins in each sideframe, and is restrained from moving freely in that direction by 2 linear springs (constant $k$ each) and dampers (constant $c$ each) at each end. In the real system this restraint is provided by the shear stiffness of the bolster springs, whose primary function is to support the car weight, and the sliding of the friction wedges laterally. Further, because the springs and dampers are separated by a distance $d$, there is a moment tending to square the truck due to both the springs (constant $4 k d^{2}$ ) and the dampers constant $4 c d^{2}$ ). In addition, the bolster has mounted symmetrically with respect to the centerplate, constant contact sidebearings (constant $\bar{k}_{B}$ each) whose function is to provide a torsional spring restraint for the bolster relative to the car body (constant $2 \bar{k}_{B} w^{2}$ ). Actually the sidebearings also transmit a damping moment between the bolster and the car body (constant $2 c_{B} w^{2}$ ); however, this has the same form as the centerplate moment and can be combined with it. There are eight degrees of freedom in this model and we will take as our independent coordinates $x^{F}, \psi^{F}, \beta^{F}, u^{F}, x^{R}, \psi^{R}, \beta^{R}, u^{R}$. Here the superscripts represent the front and rear truck coordinates, $x$ is the axial displacement of the truck centroid relative to the track center line, $\psi$ the yaw angle of each wheelset of the truck as a result of the kinematic constraint, $\beta$ the trail angle of the truck, and $u$ the bolster displacement relative to the truck centroid. The equations of motion, which have been derived elsewhere [5] and which are quite similar to others which have been discussed in the literature [4], are written here in dimensionless form in terms of sum and difference coordinates, 